known as Project Sherwood. Fuchs took a different direction. In March 1950 he was convicted of spying for Russia, and towards the end of that year CTR in the UK was classified as secret, ostensibly because of its military potential. In my view the real reason was an obsession with secrecy for its own sake, to which the United States Government was not immune, for their fusion programme was born classified in spite of concerted opposition from the scientists concerned. Even the very fact that the Atomic Energy Commission was sponsoring CTR was kept secret, as was the location of the experiments, although all three countries must have been aware of each other's interest in CTR. Surprisingly Russia was the first to publicize its fusion research through the famous Kurchatov lecture at Harwell in 1956, much to the discomfiture of the other two.

Subsequently the security barriers were gradually dismantled. First came the UKUS exchanges of information and laboratory visits. Then, after some hard bargaining in an atmosphere of rivalry and

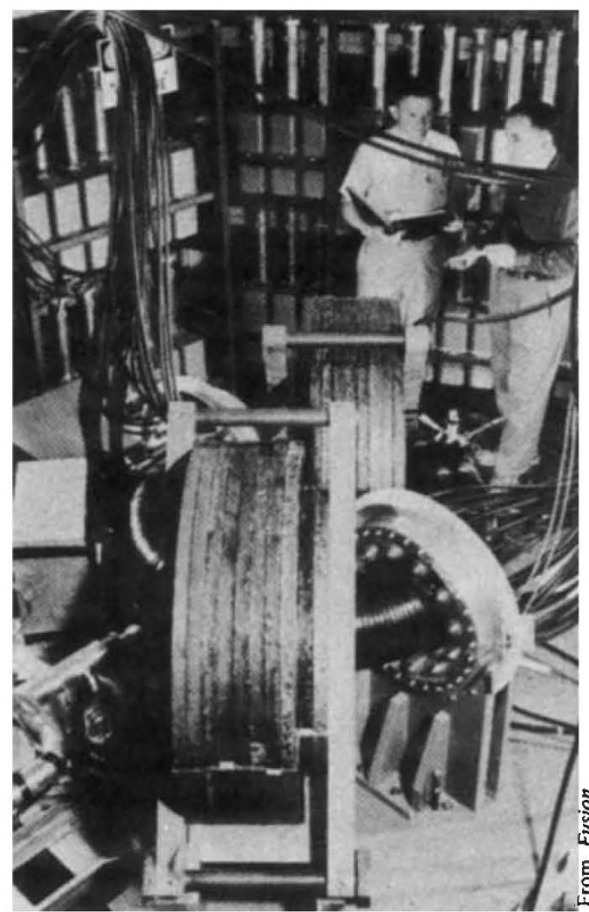

Los Alamos's Perhapsatron S-4, which together with other examples of fusion technology was displayed at the second Geneva Conference in 1958.

suspicion fomented by the Press on both sides of the Atlantic, agreement was reached on the publication in Nature in January 1958 of a group of papers which in effect formed a joint progress report on CTR. Bromberg's account of this episode may upset the more sensitive members of the Harwell team even now, and indeed it does contain some technical inaccuracies concerning ZETA, the huge toroidal gas discharge which was Britain's major fusion experiment. But in the context of a history of fusion within the United States, it is in my view a valid and objective assessment of a precarious period in UK-US collaboration.
Complete declassification of CTR was agreed in time for the second Geneva Conference in 1958, where all nations having fusion programmes would be able to reveal the extent of their progress and to exchange ideas. Here the Americans took the opportunity to mount an elaborate working exhibit, the Sherwood Spectacular, which dominated the conference and attracted 100,000 visitors. This was a great boost to US prestige, but it was only achieved at the cost of totally diverting their CTR effort for many months.

The account of the first decade of fusion work in the United States is for me the most interesting part of the book. The four government-sponsored laboratories Livermore, Los Alamos, Oak Ridge and Princeton - enjoyed a high degree of autonomy. They worked on their individual ideas with a pioneering and sometimes partisan attitude in geographical isolation; a round trip of these laboratories entailed a journey of at least 6,000 miles. This is reflected in the diversity of their early schemes for the magnetic confinement of plasma, some of which bore intriguing names. Los Alamos had its Perhapsatron and Columbus, pulsed discharges in toroidal and cylindrical tubes respectively; Livermore had magnetic mirror traps named Toy Top and Table Top; Princeton's large pretzel-shaped apparatus was called the Stellarator. Oak Ridge's direct current experiment had the more prosaic acronym, DCX. Naming fusion devices gave much scope for whimsical originality; my favourite acronym is Llandudno - large linear and no doubt unstable discharge - coined by Tony Malein at Harwell.

With the advent of international cooperation in CTR, the US experimental programme became increasingly influenced by developments overseas. Over the same period scientific direction and financial control were inexorably withdrawn from the laboratories to the central administration in Washington DC. The later chapters of the book mainly deal with the activities of this central power base under changing styles of leadership and organization. They provide an insight into the vagaries of political priorities and industrial expediency which is instructive and at times cautionary.

This book is, as far as I am aware, the first official history of a national fusion programme to be published. Earlier accounts of fusion research have in the main been written by participants rather than professional historians, and in consequence have been restricted in scope and outlook. Joan Bromberg has set high standards of sensitive objectivity, technical exposition and assiduous referencing for others to follow.

Philip Davenport joined the CTR group at Oxford in 1949 and subsequently worked on early experiments at Harwell and Culham Laboratory. Now retired, he is an honorary consultant to Culham on historical matters.

\section{Style of a physicist}

\author{
R.H. Dalitz
}

Tabibito (The Traveler).

By Hideki Yukawa.

World Scientific/Wiley: 1982. Pp.218.

$H b k £ 22.50, \$ 38 ; p b k £ 11, \$ 19$.

As a person, Hideki Yukawa was little known in Western countries, although he died only two years ago. His name, however, won international renown for his theory that the nuclear forces were mediated by a field due to massive meson, as laid out in his paper of November 1934 published in the Proceedings of the Physico-Mathematical Society of Japan, for which he received the Nobel prize in 1949. This autobiography, detailing his life from his birth in 1907 up to 1934 and telling how he came to his idea, has therefore a rather special interest.

The first surprise is to learn that Yukawa was his adoptive surname, taken when he married into the Yukawa family. His father's surname was Ogawa, but that again was an adoptive surname, for his paternal grandfather's surname was Isai. To confuse things further, his father-inlaw was born with the surname Sakabe. His father-in-law's adoption by the Yukawa family had a more understandable, although surprising reason, namely that the family Sakabe had been abolished by a local Lord, who ordered Sakabe senior to commit hara-kiri, so that Sakabe junior was in need of a family.

Despite this unfamiliar background, Yukawa's mind and its course of development conform to patterns quite familiar to us, common among physicists of imagination. The young Yukawa was strongly introverted and his inner life was the more immediate and dominant for him, until well into his late 20s. Much he considers important about his childhood goes against current educational theories. For example, his grandfather taught him the Chinese symbols by rote, introducing him to the great Chinese classics without any understanding of the text in which they were used, a training he counted a great advantage in later life. He also found great value in children's literature of an old-fashioned type which taught him important patterns of behaviour and provided models for his attitudes in life. As he put it, "the chaotic material" which came into his mind in this way "became ordered and organized into life's direction".

Yukawa had no yearning for foreign lands; his life was mostly within him. When Einstein came to Kyoto to lecture, he made no effort to attend (although he was, of course, then only in high school and did not know that he was to become a physicist). However, Yukawa's interest in modern physics was first aroused about this time, by Reiche's book on the old quantum theory and its difficulties, which he found 
"more interesting than any novel". Next, Max Planck's volumes on theoretical physics, reaching his hand by chance, conveyed the essentials of the subject to him in a clear and direct way, despite their being in German. His first awareness that physics was on the move at last, came from a lecture given by Professor Nagaoka in a visit to Kyoto during Yukawa's first year as a university student and Schrödinger's papers on quantum mechanics became the young Yukawa's bible in the following year; wisely, he read only from the original papers and came to know them through and through.

Yet, even so, Yukawa felt that he came into theoretical physics, not through conscious choice, but by the exclusion of other possibilities where his personality and intuition gave him no advantage, until theoretical physics was the door most readily open to him. His first postgraduate years were spent as an unpaid research associate in the Tamaki research room at Kyoto University. In 1929, when Heisenberg and Pauli made their famous trip around the world, they came to Kyoto and their lectures were an immense stimulus to the young Yukawa. S. Tomonaga was a fellow student through this period, "an outstanding companion . . . encouraging and challenging .... the type of person who is aware of limitations and yet comes up with the clever ideas".

In 1932, the discovery of the neutron, the positron and nuclear disintegration directed Yukawa's attention to the nucleus and the forces which held it together. Psychologically the time was ripe for him. Nishina had come to Kyoto about 1930 , after a long stay with Niels Bohr at Copenhagen, and in his presence "my solitary mind began to open". Around this time he married Sumi Yukawa, whose photograph had attracted him instantly when a gobetween came to his father (Ogawa) proposing marriage. This moved him outside his family circle and the young couple set up house in Osaka, a neighbouring city, as open and lively a city as Kyoto was closed, private and quiet. He was then appointed to a lectureship at Kyoto; Sakata and Kobayashi were students at his first lectures and Taketani came soon after, and they, together with Tomonaga (by then in Tokyo), became his close friends. Next, a university was established at Osaka and he became a lecturer in Professor Yagi's department there; a happy situation, since he felt great admiration for Yagi.

Through this period, Yukawa's work with the nuclear force problem went on almost subconsciously. Already in 1932, he had reached the conviction that there must be some new force field involved but, as he said, "the path thereafter was not straight: I had to take the wrong path first, before I could arrive at my destination'". Then, one night in October 1934, the crucial point occurred to him that the range of nuclear forces must be connected with the mass of the new particle he was seeking. Next morning he calculated its mass to be about $100 \mathrm{MeV}$. The particle had not been seen experimentally since such high energies were not available in the laboratory. $\mathrm{He}$ quickly became confident and predicted that such particles should be found in cosmic rays, in Wilson cloud-chamber experiments. He presented his new theory to the Physico-Mathematical Society of Japan, but his wife kept urging "please

\section{IMAGE UNAVAILABLE FOR COPYRIGHT REASONS}

write the English paper and show it to the world"'; so, an English-language paper was quickly sent to the Society for publication.

Young physicists today find it difficult to understand the mental attitudes of physicists even 30 years ago. They wonder why it took so long for physicists of Yukawa's time to propose a new particle to solve this problem. The fact is that to hypothesize unobserved particles to account for new phenomena was disreputable in those days; physicists were conservative and progress in physics was not to be made in that way. Evidence of a particle was needed first; then we could talk about its properties and purpose! Even in 1954, John Wheeler gave "a sermon" to the American Physical Society with the text from St John: "Believe in those things which are good", urging us to explore thoroughly the established theories of electromagnetism and gravitation, before invoking new physical interactions to account for the elementary particles then being discovered. The resulting work led to many unexpected and important results, although it achieved little towards our understanding of these particles. Today, a theoretician feels no embarrassment in proposing a new theory involving several dozen unknown particles, nor in proposing some different theory in the following month. This was not Yukawa's style.

Yukawa's autobiography, written in his fiftieth year, is not concerned with his later work, and this helps to focus our attention on the importance of quiet reflection and subconscious thought on basic problems. We may quote a poem Yukawa once wrote:

\section{In Kamakura,}

here in a deep and quiet valley, a man walks deep in thought.

He had in mind a particular professor of philosophy, who impressed him greatly, but we may take it as Yukawa's epitaph. $\square$

R.H. Dalitz is Royal Society Research Professor at the University of Oxford.

\section{Man and machine}

\section{Brian Gaines and Mildred Shaw}

\section{Micro Man: Living and Growing with Computers.}

By Gordon Pask and Susan Curran. Century, 76 Old Compton St, London: 1982. Pp.222. £8.95.

TO LIVE a life unaffected by computers is becoming increasingly difficult. Soon it may be impossible. Whether the prospect excites or appalls you could well be shaped by reading this book. The medium of print has been used to present the message vividly with pictures and graphics on every page, many in colour. An erudite text has been transformed through brilliant layout and illustration into an everyman's guide to computers. Not only are the techniques here - you can use the book as a guide to chips and bytes, Apples and COBOLs but also the intuitive feel for the culture.

Gordon Pask should need little introduction. $\mathrm{He}$ is an authentic genius, a visionary yet an immensely practical man, whose cybernetic theories have influenced many in engineering, psychology, education and the arts. Pask built personal computers before the name was coined. Some were intended to interact with people and others to be people. During a quartercentury of intellectual innovation he has used the technology of the day to create models of, and tools for investigating, a range of theories that encompass questions going to the roots of life itself. If anyone can speak for both humanity and computers it is Pask.

Susan Curran brings to the collaboration professional experience of presenting advances in electronic, computer and office technology to businessmen and others concerned with their economic and social impact. Her partnership with Pask has resulted in a lucid book that illuminates the often obfuscated issues of computers and their impact on us, our societies and ourselves.

This is a book of questions rather than answers - it probes and it speculates and it wonders. Many of the questions are profoundly disturbing, not only because we do not know the answers, but also because many of those that we dimly perceive are unacceptable and frightening. Modern 